\title{
Rats counting rats: The use of conspecifics as discriminative stimuli
}

\author{
HANK DAVIS and LAURIE HIESTAND \\ University of Guelph, Guelph, Ontario, Canada
}

\begin{abstract}
Although a variety of experimental procedures have shown considerable evidence of "counting" in animals, there is no evidence of numerical competence when conspecifics are used as test stimuli. Here, we report evidence of a relative numerousness ( $2 \mathrm{vs} .4$ ) judgment by rats that were required to discriminate the number of free-ranging conspecifics in an arena. This discrimination was ultimately disrupted by vigorous social interaction between the subjects and the to-becounted rats. Attempts to restrict the movement of object rats in Experiment 2 did not lead to reestablishment of the discrimination, although 2 animals did show evidence of a simple presence versus absence ( 0 vs. 5 ) discrimination, a precursor of numerical ability.
\end{abstract}

Extensive research indicates that rats can use the numerical attribute of stimuli as a discriminative cue for performance in a variety of situations (Davis \& Memmott, 1982; Davis \& Pérusse, 1988b). For example, rats have been required to discriminate the number of food pellets (Capaldi \& Miller, 1988; Davis \& Bradford, 1991), bursts of white noise (Davis \& Albert, 1986; Fernandes \& Church, 1982), the ordinal position of tunnels (Davis \& Bradford, 1986), and the number of strokes delivered to the vibrissae (Davis, MacKenzie, \& Morrison, 1989).

Although these studies have demonstrated that a wide range of stimuli can serve as the basis for numerical control, none has determined whether a rat can use the number of other rats present in a situation as a discriminative cue. Because rats live and forage in social groups of varying sizes (Barnett, 1963), it is reasonable to investigate whether they can perform differentially on the basis of the number of other rats in a group.

In a related study, Husted and McKenna (1966) reported that rats were able to discriminate between the presence and absence of conspecifics, and could use this information to determine whether or not leverpressing would be reinforced. The detection of presence versus absence is a precursor of numerical ability (Davis \& Pérusse, 1988b). In the present experiments, we attempted to demonstrate the most rudimentary form of numerical competence: the distinction between many and few. This ability, termed a relative numerousness judgment (Davis \& Pérusse, $1988 \mathrm{~b}$ ), has been established in a variety of nonsocial settings (e.g., Davis et al., 1989; Fernandes \& Church, 1982). Here, we tested whether rats could discriminate

This research was supported in part by Grant A0673 from the National Sciences and Engineering Research Council of Canada to the first author. The authors are indebted to Craig Gallacher for his technical assistance. Correspondence should be addressed to H. Davis, Department of Psychology, University of Guelph, Guelph, ON, Canada N1G 2W1. between the presence of many (4 or 5) versus few (1 or 2) conspecifics.

Precautions to prevent subjects from detecting the presence of particular animals were taken by randomly selecting "object rats" (i.e., those to be counted) from a pool of up to 30 individuals.

\section{EXPERIMENT 1}

In Experiment 1, the subjects were placed in an enclosure along with either 2 or 4 free-ranging conspecifics and required to leave the arena using either a left or right doorway, depending on the number of other rats present in the enclosure (e.g., if 2, go left; if 4, go right).

\section{Method}

Subjects. Six female Long-Evans rats (Rattus norvegicus), approximately 10 months old, served as subjects. The animals were maintained at $90 \%-95 \%$ of ad-lib weight. In addition, 30 rats of the same strain, sex, and approximate age were used as object rats. These animals were not food-deprived. All rats were housed individually in $24 \times 18 \times 18.5 \mathrm{~cm}$ wire cages and were allowed unlimited access to water.

Apparatus. The subjects were tested in a $1.5 \times 0.9 \mathrm{~m}$ arena, which had a startbox $(29 \times 17.5 \times 24.5 \mathrm{~cm})$ centered at one end. A guillotine door allowed access from the startbox to the arena. The walls of the plywood enclosure were $32 \mathrm{~cm}$ high, and the floor was covered with a layer of granulated corncob to a depth of approximately $5 \mathrm{~cm}$. Two wooden tunnels were placed at the far end of the arena, opposite the startbox. Each tunnel was $22 \times 10 \times 8.9 \mathrm{~cm}$, and could be entered through a hinged door that swung freely unless restricted by a $20-\mathrm{cm}$-long plywood insert. When restricted, the tunnel door could only swing open about $2 \mathrm{~cm}$, thus preventing access. Both tunnels were baited with a single flake of sugar-frosted cereal.

Procedure. Subject rats were initially trained over a period of 15 days to enter both tunnels to receive food. At the beginning of every trial, a subject rat was placed in the startbox. The trial was begun when the guillotine door of the startbox was raised. Once right-left training had been initiated, a choice was recorded as soon as the subject either entered the correct tunnel or nudged the incorrect tunnel door with its nose. Both tunnels were baited on every trial, although the wooden insert prohibited access to food in the incorrect tunnel.

When the subject made a correct response, it gained immediate access to the food. After consuming the reward, it was removed to the holding area where it was provided with another cereal flake while the 
Table 1

Number of Correct Responses per Blocks of 25 Trials in Experiment 1

\begin{tabular}{ccccccc}
\hline \multirow{2}{*}{$\begin{array}{c}\text { Trial } \\
\text { Block }\end{array}$} & 1 & 2 & 3 & 4 & 5 & 6 \\
\hline \cline { 2 - 7 } & 15 & $17^{*}$ & $21^{*}$ & $20^{*}$ & 13 & 14 \\
2 & 10 & 16 & $17^{*}$ & 14 & 14 & 16 \\
3 & 10 & 15 & 15 & $17^{*}$ & 11 & $21^{*}$ \\
4 & 15 & 10 & 8 & 12 & 13 & 14 \\
Average & 12.50 & 14.50 & 15.25 & 15.75 & 12.75 & 16.25 \\
\hline
\end{tabular}

${ }^{*} p<.05$.

arena was set up for the next trial. When the subject made an incorrect choice and found that the tunnel door was blocked, the experimenter clapped once and said "No!" The rat was then removed to a holding area that prevented it from seeing the enclosure. This "time out" remained in effect for several minutes while the arena was prepared for the next trial. Between trials, the experimenter raked the bedding material to disrupt scent markings.

Two of the subjects (Subjects 3 and 6) were randomly selected to be reinforced for entering the left tunnel when 2 object rats were present, and the right tunnel when 4 object rats were present. The remaining animals were trained under the opposite requirement (i.e., 2 object rats, right tunnel; 4 , left tunnel). The selection of 2 versus 4 object rats in the enclosure varied randomly over trials within a session. Individual object rats were chosen so that no animal appeared more frequently than once per session for each subject. Moreover, individual object rats appeared in both the " 2 " and " 4 " conditions across sessions, thereby precluding correct performance based on the recognition of particular individual rats. When not required for a trial, the object rats were caged communally. After completing a trial, each object rat was moved to a second communal chamber where it was held until a new subject was run.

Subject rats were given five trials per daily session; sessions were run 6 days per week. One hundred trials were presented in Experiment 1, during which either 2 or 4 object rats were allowed to roam unrestrained in the test arena. All sessions were videotaped.

Data analysis. Performance was analyzed in blocks of 25 trials. The criterion for successful performance was determined by a binomial probability table. A minimum of $17 / 25$ correct responses was required for a significant $(p<.05)$ trial block.

\section{Results}

The data for Experiment 1 are summarized in Table 1. Four of 6 subjects were successful on at least one trial block in discriminating the presence of 2 versus 4 object rats in the enclosure and in using this information as the basis for correct performance. Three of these subjects reached criterion during the initial trial block, but as they became more involved in social interactions with object rats, their performance deteriorated. In only 1 subject (Subject 6) was there evidence of a "learning curve," that is, a progressive improvement in performance over trials, although even this animal reverted to its lowest level of performance during the final block of trials.

In general, vigorous social interaction between the subject rat and the free-ranging object rats had a progressively negative effect on performance. Even in cases where the subject did not instigate social contact, it was often the target of interactions by one or more of the object animals. Moreover, because group membership changed on a random basis, exploratory behavior typical of initial encounters continued to occur and yielded no reduction in the amount of disruptive social behavior over sessions. In- formal observation suggested that performance was most successful on trials in which the subject was not distracted by social interaction with object rats.

\section{Discussion}

Although numerical discrimination does appear attainable under these conditions, our decision to allow object rats to range freely in the test arena produced extensive social disruption of performance. We were therefore unable to determine the extent to which the number of conspecifics could serve as a discriminative cue for performance. This condition was modified in Experiment 2.

\section{EXPERIMENT 2}

The presence of a freely interacting conspecific may be a sufficiently salient stimulus to detract from a subject's attention or motivation to perform in tests of numerical competence. There is considerable evidence that rats are highly social, even within the relatively sterile confines of experimental testing. For example, Davis and Pérusse (1988a) demonstrated that rats could be trained to leverpress in order to receive social contact with a human. In addition, Latané, Joy, Meltzer, Lubell, and Cappell (1972) found that rats preferred to approach conspecifics that were able to interact with them rather than to approach anesthetized or restrained rats. Similarly, Humphreys and Einon (1981) reported that rat subjects chose unrestrained rats over drugged or caged rats in a T-maze.

In Experiment 2, we attempted to minimize the disruptive social influence of free-ranging object rats. The subjects were again required to perform a relative numerousness judgment between 2 and 4 conspecifics; however, object rats were confined to individual wire-mesh cages located within the test arena. In addition to retesting previously successful subjects from Experiment 1, experimentally naive animals were exposed to the modified test procedure in Experiment 2.

\section{Method}

Subjects. Subjects 1-6, tested in Experiment 1, also served as subjects in Experiment 2. In addition, 4 naive animals (Subjects 7-10) were tested. The pool of 30 object rats remained the same.

Apparatus. The arena used in Experiment 2 was identical to that used in Experiment 1 . The object rats were restrained individually in $22 \times 8 \times$ $8 \mathrm{~cm}$ wire-mesh cages (1-cm-sq mesh) with shellacked wooden floors.

Procedure. The procedure used in Experiment 1 was also followed in Experiment 2, with the exception that the object rats were no longer allowed to move freely around the test arena. Instead, they were placed in individual wire cages, which allowed the subject rats to investigate them, but which precluded more intense interaction. Six cages were positioned in the arena, three on each side, leaving a central alley for the subject rat to move between them. This procedure was informally termed "rat at the zoo," since it encouraged subject rats to visit individual cage locations in order to discriminate numerical conditions. In the case of Subjects 1-6, either 2 or 4 object rats were placed randomly within these six cages. The naive subjects (Subjects 7-10) were tested on a putatively easier discrimination between 1 and 5 object rats in Trial Block 1 , and switched to the simplest 0 versus 5 discrimination in the final trial block.

As in Experiment 1, object rats were selected so that no animal was used more than once per day for each subject. The wire cages were wiped to remove urine and feces when necessary, and were rinsed at the end of every day. The subjects were run for a total of $\mathbf{5 0}$ trials. Sub- 
Table 2

Number of Correct Responses per Blocks of 25 Trials in Experiment 2

\begin{tabular}{ccccccccccc}
\hline \multirow{2}{*}{$\begin{array}{c}\text { Trial } \\
\text { Block }\end{array}$} & 1 & 2 & 3 & 4 & 5 & 6 & 7 & 8 & 9 & 10 \\
\hline 1 & 12 & 12 & 14 & 14 & 13 & 11 & 12 & 13 & 12 & 13 \\
2 & 10 & 14 & 15 & 15 & 12 & 13 & $18 *$ & 13 & $19 *$ & 14 \\
\hline
\end{tabular}

Note-Subjects 1-6 were tested with 2 versus 4 discrimination. Subjects 7-10 were required to discriminate between 1 versus 5 animals during Trial Block 1, and 0 versus 5 animals in Trial Block $2 .{ }^{*} p<.05$.

jects 7 and 9 were required to exit left in the presence of 1 or 0 object rats and to exit right when 5 animals were present. Subjects 8 and 10 were exposed to the opposite requirement.

\section{Results}

The performance of all subjects is summarized in Table 2. Despite minimizing the amount of social interaction with object rats, none of the previously trained subjects reached significant performance on either trial block. Similarly, the naive subjects showed no evidence of making the relatively simple 1 versus 5 discrimination when tested during Trial Block 1. Two subjects (Subjects 7 and 9) did show significant performance when the numerical requirement was reduced to a presence versus absence (0 vs. 5) discrimination in Trial Block 2.

Despite the use of cages, the subjects generally approached and attempted to interact with the restrained conspecifics, as they had in Experiment 1. There was considerable oral contact by both subject rats and object rats through the wire-mesh enclosure. One subject (Subject 2) temporarily established a pattern of urinating on each object rat it visited, thus suggesting the possibility that performance would involve "partitioning and tagging," an essential component of enumeration. Unfortunately, this pattern did not remain in effect long enough to be evaluated systematically.

\section{GENERAL DISCUSSION}

Although there is considerable evidence that rats are capable of different forms of numerical competence (see reviews by Davis \& Memmott, 1982; Davis \& Pérusse, 1988b), the evidence of numerical competence using social stimuli is considerably less developed. Husted and McKenna (1966) demonstrated a precursor to numerical ability by showing that rats could use the presence versus absence of other rats as a discriminative cue for performance. We have replicated this effect here in 2 animals during the final trial block of Experiment 2.
Unfortunately, our attempts to demonstrate a relative numerousness judgment were only marginally successful. In Experiment 1, rats quickly learned to discriminate the presence of 2 versus 4 conspecifics, although interactions with the enumerated animals ultimately led to disruption of performance. The physical restriction of object rats within the test arena did not allow the more versus less numerical discrimination to reemerge in Experiment 2, nor did it result in discriminative learning in naive animals.

In short, although there is considerable evidence that rats are capable of making numerical discriminations under a variety of settings, the social arena does not appear to be an ideal setting for such demonstrations. The present data suggest that numerical discrimination of conspecifics may work best when using highly interactive animals, the very presence of which ultimately undermines the motivation and attention necessary to perform the task.

\section{REFERENCES}

Barnett, S. A. (1963). A study in behavior. Chicago: Aldine.

Capaldi, E. J., \& Miller, D. J. (1988). Counting in rats: Its functional significance and the independent cognitive processes that constitute it. Journal of Experimental Psychology: Animal Behavior Processes, 14, 3-17.

Davis, H., \& Albert, M. (1986). Numerical discrimination by rats using sequential auditory stimuli. Animal Learning \& Behavior, 14, 57-59.

Davis, H., \& Bradford, S. A. (1986). Counting behavior by rats in a simulated natural environment. Ethology, 73, 265-280.

DAVIS, H., \& BRADFORD, S. A. (1991). Numerically restricted food intake in the rat in a free-feeding situation. Animal Learning \& Behavior, 19, 215-222.

Davis, H., MacKenzie, K., \& Morrison, S. (1989). Numerical discrimination using body and vibrissal touch in the rat. Journal of Comparative Psychology, 103, 45-53.

Davis, H., \& MEмmotT, J. (1982). Counting behavior in animals: A critical evaluation. Psychological Bulletin, 92, 547-571.

DAvis, H., \& PÉRUSSE, R. (1988a). Human-based social interaction can reward a rat's behavior. Animal Learning \& Behavior, 16, 89-92.

DAVIS, H., \& PÉRUSSE, R. (1988b). Numerical competence in animals: Definitional issues, current evidence and a new research agenda. $B e-$ havioral \& Brain Sciences, 11, 561-615.

Fernandes, D. M., \& ChURCh, R. (1982). Discrimination of the number of sequential events by rats. Animal Learning \& Behavior, 10, 171-176.

Humphreys, A. P., \& EinON, D. F. (1981). Play as a reinforcer for maze-learning in juvenile rats. Animal Behavior, 29, 259-270.

Husted, J. R., \& MCKENNA, F. S. (1966). The use of rats as discriminative stimuli. Journal of the Experimental Analysis of Behavior, 9, 677-679.

latané, B., Joy, V., Meltzer, J., Lubell, B., \& Cappell, H. (1972). Stimulus determinants of social attraction in rats. Journal of Comparative \& Physiological Psychology, 79, 13-21.

(Manuscript received April 16, 1992.) 